\title{
Gold-Enhanced Biomolecular Surface Imaging of Cells and Tissue by SIMS and MALDI Mass Spectrometry
}

\author{
A. F. Maarten Altelaar, ${ }^{\dagger}$ Ivo Klinkert, ${ }^{\dagger}$ Kees Jalink, ${ }^{\ddagger}$ Robert P. J. de Lange, $\$$ Roger A. H. Adan, $\$$ \\ Ron M. A. Heeren, ${ }^{\dagger}$ and Sander R. Piersma*,
}

FOM Institute for Atomic and Molecular Physics, Kruislaan 407, 1098 SJ Amsterdam, The Netherlands, Division of Cell Biology, Netherlands Cancer Institute, Plesmanlaan 121, 1066 CX Amsterdam, The Netherlands, and Department of Pharmacology and Anatomy, Rudolf Magnus Institute of Neuroscience, University Medical Center Utrecht, Universiteitsweg 100, 3584 CG, Utrecht, The Netherlands

Surface metallization by plasma coating enhances desorption/ionization of membrane components such as lipids and sterols in imaging time-of-flight secondary ion mass spectrometry (TOF-SIMS) of tissues and cells. High-resolution images of cholesterol and other membrane components were obtained for neuroblastoma cells and revealed subcellular details (resolving power $1.5 \mu \mathrm{m}$ ). Alternatively, in matrix-enhanced SIMS, 2,5-dihydroxybenzoic acid electrosprayed on neuroblastoma cells allowed intact molecular ion imaging of phosphatidylcholine and sphingomyelin at the cellular level. Gold deposition on top of matrix-coated rat brain tissue sections strongly enhanced image quality and signal intensity in stigmatic matrixassisted laser desorption/ionization imaging mass spectrometry. High-quality total ion count images were acquired, and the neuropeptide vasopressin was localized in the rat brain tissue section at the hypothalamic area around the third ventricle. Although the mechanism of signal enhancement by gold deposition is under debate, the results we have obtained for cells and tissue sections illustrate the potential of this sample preparation technique for biomolecular surface imaging by mass spectrometry.

Unraveling the spatial distribution of cellular membrane components is an important research topic in current molecular cell biology. Understanding the behavior and function of the major constituents of these membranes, i.e., lipids and sterols, has been hampered by methodological limitations, despite their relatively simple structures. Most of the current knowledge on lipid localization has been obtained using fluorescence imaging techniques. ${ }^{1-4}$ The drawback of this technique is the need for fluorescent labeling of the molecule of interest. This is especially

\footnotetext{
* Corresponding author. Tel: +31-20-6081394. Fax: +31-20-6684106. E-mail: piersma@amolf.nl.

$\dagger$ FOM Institute for Atomic and Molecular Physics.

₹ Netherlands Cancer Institute.

$\S$ Rudolf Magnus Institute of Neuroscience.

(1) Huster, D.; Muller, P.; Arnold, K.; Herrmann, A. Biophys. J. 2001, 80, 822831.

(2) Zhang, J.; Campbell, R. E.; Ting, A. Y.; Tsien, R. Y. Nat. Rev. Mol. Cell Biol. 2002, 3, 906-918.

(3) Maier, O.; Oberle, V.; Hoekstra, D. Chem. Phys. Lipids 2002, 116, 3-18.
}

difficult in the case of lipids where only a few anti-lipid antibodies or specific lipid-binding protein domains ${ }^{5}$ are available for fluorescent readout. Artificial fluorescent probes are used that completely replace one of the fatty acid chains, ${ }^{3,4}$ potentially resulting in perturbation of the lipid (physico) chemical properties..$^{3-5}$

Ideally, a molecular imaging method should image the distribution of the native membrane components, omitting labeling altogether. One of the emerging alternatives in this field is imaging mass spectrometry, which provides both chemical specificity and spatial distributions of native components present at a bioorganic surface. ${ }^{6}$ Recently, imaging matrix-assisted laser desorption/ ionization mass spectrometry (MALDI-MS) ${ }^{7}$ has been recognized as a tool for in situ proteomic tissue analysis. ${ }^{8,9}$ Imaging MALDIMS can typically analyze molecules up to $100 \mathrm{kDa}$ but has a limited spatial resolution of $\sim 30-100 \mu \mathrm{m}^{10,11}$ Secondary ion mass spectrometry (SIMS) can routinely achieve submicrometer spatial resolution, ${ }^{12}$ using primary ion beams such as $\mathrm{Ga}^{+}$. However, the sensitivity for high-mass ions is limited due low secondary ion yields ${ }^{13}$ as well as low detection efficiencies in current SIMS instrumentation. ${ }^{14}$ SIMS has been used for imaging of elements (Na, K, Ca, etc.) in cells at high spatial resolution $(\sim 50 \mathrm{~nm})^{15,16}$ and in molecular imaging of (freeze fractured) single cells, showing only specific lipid fragments such as the phosphocholine (PC) headgroup at $m / z 184$ Da. ${ }^{17-20}$

(4) Kuerschner, L.; Ejsing, C. S.; Ekroos, K.; Shevchenko, A.; Anderson, K. I.; Thiele, C. Nat. Methods 2005, 2, 39-45.

(5) Ishitsuka, R.; Yamaji-Hasegawa, A.; Makino, A.; Hirabayashi, Y.; Kobayashi, T. Biophys. J. 2004, 86, 296-307.

(6) Stoeckli, M.; Chaurand, P.; Hallahan, D. E.; Caprioli, R. M. Nat. Med. 2001 , 7, 493-496.

(7) Caprioli, R. M.; Farmer, T. B.; Gile, J. Anal. Chem. 1997, 69, 4751-4760.

(8) Hanash, S. Nature 2003, 422, 226-232.

(9) Aebersold, R.; Mann, M. Nature 2003, 422, 198-207.

(10) Kruse, R.; Sweedler, J. V. J. Am. Soc. Mass Spectrom. 2003, 14, 752-759.

(11) Chaurand, P.; Schwartz, S. A.; Caprioli, R. M. Anal. Chem. 2004, 76, 86a-93a.

(12) Todd, P. J.; McMahon, J. M.; Short, R. T.; McCandlish, C. A. Anal. Chem. 1997, 69, A529-A535.

(13) Adriaensen, L.; Vangaever, F.; Lenaerts, J.; Gijbels, R. Rapid Commun. Mass Spectrom. 2005, 19, 1017-1024.

(14) Gilmore, I. S.; Seah, M. P. Int. J. Mass Spectrom. 2000, 202, 217-229.

(15) Chandra, S.; Smith, D. R.; Morrison, G. H. Anal. Chem. 2000, 72, 104A$114 \mathrm{~A}$.

(16) Strick, R.; Strissel, P. L.; Gavrilov, K.; Levi-Setti, R. J. Cell Biol. 2001, 155, 899-910.

10.1021/ac0513111 CCC: $\$ 33.50$ @ 2006 American Chemical Society Published on Web 12/20/2005 
In conventional SIMS analysis, a very thin (mono)layer of sample is deposited on a surface. To enhance the ionization yield for large intact molecular ions by SIMS, different kinds of surface modifications (MALDI matrixes, ${ }^{21-25}$ silver ${ }^{26}$ and gold ${ }^{27-31}$ ) as well as the use of polyatomic primary ion beams ${ }^{32-37}$ have been reported. Metallization of (organic) samples with silver and gold has been demonstrated to increase secondary ion yields of intact molecular ions in SIMS, ${ }^{26-31}$ this technique is named metalassisted (MetA) SIMS. The exact mechanism leading to the enhanced sputter yields of organic species after metallization is still under debate. Several explanations have been proposed: First, there is migration of mobile analytes onto gold nanoislands. ${ }^{38}$ Second, gold enhances desorption/ionization by cooperative elevation of analyte molecules and metal atoms with similar momentum, as shown by molecular dynamics simulations. ${ }^{39}$ Third, gold acts as cationizing agent leading to improved ion formation. ${ }^{28,40}$ Additionally, suppression of surface charging by the presence of gold has shown to improve the quality of the images obtained for insulating samples. ${ }^{29}$

Another way of modifying the bioorganic surface is deposition of a MALDI matrix. In 1996, Wu and Odom ${ }^{21}$ showed that MALDI sample preparation protocols can be used in SIMS to enhance the desorption/ionization yield of "large" molecular species. This

(17) Colliver, T. L.; Brummel, C. L.; Pacholski, M. L.; Swanek, F. D.; Ewing, A. G.; Winograd, N. Anal. Chem. 1997, 69, 2225-2231.

(18) Roddy, T. P.; Cannon, D. M.; Meserole, C. A.; Winograd, N.; Ewing, A. G. Anal. Chem. 2002, 74, 4011-4019.

(19) Roddy, T. P.; Cannon, D. M.; Ostrowski, S. G.; Winograd, N.; Ewing, A. G. Anal. Chem. 2002, 74, 4020-4026.

(20) Ostrowski, S. G.; Van Bell, C. T.; Winograd, N.; Ewing, A. G. Science 2004 305, 71-73

(21) Wu, K. J.; Odom, R. W. Anal. Chem. 1996, 68, 573-882.

(22) Luxembourg, S. L.; McDonnell, L. A.; Duursma, M. C.; Guo, X. H.; Heeren, R. M. A. Anal. Chem. 2003, 75, 2333-2341.

(23) Wittmaack, K.; Szymczak, W.; Hoheisel, G.; Tuszynski, W.J. Am. Soc. Mass Spectrom. 2000, 11, 553-563.

(24) Altelaar, A. F. M.; van Minnen, J.; Jimenez, C. R.; Heeren, R. M. A.; Piersma, S. R. Anal. Chem. 2005, 77, 735-741.

(25) McDonnell, L. A.; Piersma, S. R.; Altelaar, A. F. M.; Mize, T. H.; Luxembourg, S. L.; Verhaert, P. D. E. M.; van Minnen, J.; Heeren, R. M. A. J. Mass Spectrom. 2005, 40, 160-168.

(26) Nygren, H.; Malmberg, P.; Kriegeskotte, C.; Arlinghaus, H. F. FEBS Lett. 2004, 566, 291-293.

(27) Delcorte, A.; Medard, N.; Bertrand, P. Anal. Chem. 2002, 74, 4955-4968.

(28) Delcorte, A.; Bour, J.; Aubriet, F.; Muller, J. F.; Bertrand, P. Anal. Chem. 2003, 75, 6875-6885.

(29) Delcorte, A.; Bertrand, P. Appl. Surf. Sci. 2004, 231-2, 250-255.

(30) Keune, K.; Boon, J. J. Surf. Interface Anal. 2004, 36, 1620-1628.

(31) Adriaensen, L.; Vangaever, F.; Gijbels, R. Anal. Chem. 2004, 76, 67776785.

(32) Townes, J. A.; White, A. K.; Wiggins, E. N.; Krantzman, K. D.; Garrison, B. J.; Winograd, N. J. Phys. Chem. A 1999, 103, 4587-4589.

(33) Nguyen, T. C.; Ward, D. W.; Townes, J. A.; White, A. K.; Krantzman, K. D.; Garrison, B. J. J. Phys. Chem. B 2000, 104, 8221-8228.

(34) Weibel, D.; Wong, S.; Lockyer, N.; Blenkinsopp, P.; Hill, R.; Vickerman, J. C. Anal. Chem. 2003, 75, 1754-1764.

(35) Touboul, D.; Halgand, F.; Brunelle, A.; Kersting, R.; Tallarek, E.; Hagenhoff, B.; Laprevote, O. Anal. Chem. 2004, 76, 1550-1559.

(36) Sjovall, P.; Lausmaa, J.; Johansson, B. Anal. Chem. 2004, 76, 4271-4278

(37) Todd, P. J.; McMahon, J. M.; McCandlish, C. A. J. Am. Soc. Mass Spectrom. 2004, 15, 1116-1122.

(38) Hagenhoff, B. In ToF-SIMS: surface analysis by mass spectrometry; Vickerman, J. C., Briggs, D., Eds.; SurfaceSpectra and IM: Chichester, 2001; p 293.

(39) Delcorte, A.; Vanden Eynde, X.; Bertrand, P.; Vickerman, J. C.; Garrison, B. J. J. Phys. Chem. B 2000, 104, 2673-2691.

(40) Benninghoven, A.; Rüdenauer, F. G.; Werner, H. W. Secondary ion mass spectrometry: basic concepts, instrumental aspects, applications, and trends; J. Wiley: New York, 1987 technique is named matrix-enhanced (ME)-SIMS. In MALDI, the presence of a matrix results in less fragmentation of the molecular ions by removal of most of the incident beam energy and collisional cooling of the ions in the plume with the matrix molecules. ${ }^{41}$ We recently showed the usefulness of ME-SIMS in direct molecular imaging of biological tissue at subcellular resolution ${ }^{24,25}$ as well as in the analysis of phospholipids. ${ }^{22}$ Here we compare ME-SIMS and MetA-SIMS in the direct molecular analysis of single neuroblastoma cells and rat brain tissue sections. Both techniques deliver high-resolution images of different molecular distributions at cellular resolution, without the need to label the target molecules. In addition, we show that gold coating of matrix sprayed rat brain tissue sections results in improved MS image quality of neuropeptide distributions in stigmatic MALDI imaging MS.

\section{EXPERIMENTAL SECTION}

Materials. 2,5-Dihydroxybenzoic acid (DHB), $\alpha$-cyano-4-hydroxycinnamic acid (HCCA), trifluoroacetic acid (TFA), HPLC grade water, sucrose, cholesterol, and PC brain extract were purchased from Sigma-Aldrich (Zwijndrecht, The Netherlands). Ethanol and methanol were purchased from Biosolve (Valkenswaard, The Netherlands).

Cell Cultures. Neuroblastoma cells were seeded in six-well plates at $\sim 25000$ cells/well on conductive indium tin oxide (ITO)coated glass slides (Delta Technologies, Stillwater, MN) and cultured in $3 \mathrm{~mL}$ of DMEM (Invitrogen, Breda, The Netherlands) supplemented with $10 \%$ serum and antibiotics. The cells were washed in $300 \mathrm{mM}$ sucrose solution and Milli-Q water after which they were frozen on dry ice, freeze-dried for $30 \mathrm{~min}$, and stored at $-80{ }^{\circ} \mathrm{C}$. Prior to mass spectrometry, the cell cultures were brought to room temperature in a desiccator over a silica gel canister $(1 \mathrm{~h})$. Conservation of cell morphology was checked by optical microscopy, using a Leica DMRX microscope with a Nikon DXM1200 digital camera.

Tissue Sections. Experimental procedures were in accordance with the European directives (86/609/EEC) and approved by the Commission on Laboratory Animal Experiments of the University Medical Centre Utrecht. Male Wistar rats (Crl:WU) weighing $350 \mathrm{~g}$ were obtained from Charles River. Rats were decapitated without prior anesthesia, and brains were dissected and frozen in liquid isopentane, cooled to $-50{ }^{\circ} \mathrm{C}$ on dry ice, and then stored at $-80^{\circ} \mathrm{C}$ until sectioning. The $10-\mu$ m-thick rat brain tissue sections were cut at Interaural $7.2 /$ Bregma $-1.8 \mathrm{~mm},{ }^{42}$ using a cryomicrotome. Sections were thaw-mounted on ITOcoated glass slides and were stored at $-80^{\circ} \mathrm{C}$ until use. Prior to mass spectrometry, tissue sections were brought to room temperature in a desiccator over a silica gel canister $(1 \mathrm{~h})$. For stigmatic MALDI experiments, tissues were briefly washed in cold $70 \%$ ethanol and dried at room temperature before matrix deposition. ${ }^{43}$

Matrix Deposition. ME-SIMS matrix was applied using electrospray deposition as described previously. ${ }^{44,45}$ Briefly, a 15

(41) Zenobi, R.; Knochenmuss, R. Mass Spectrom. Rev. 1998, 17, 337-366.

(42) Paxinos, G.; Watson, C. The rat brain in stereotaxic coordinates, 4th ed.; Academic Press: San Diego, 1998.

(43) Schwartz, S. A.; Reyzer, M. L.; Caprioli, R. M. J. Mass Spectrom. 2003, 38 , 699-708.

(44) Hensel, R. R.; King, R. C.; Owens, K. G. Rapid Commun. Mass Spectrom. 1997, 11, 1785-1793. 

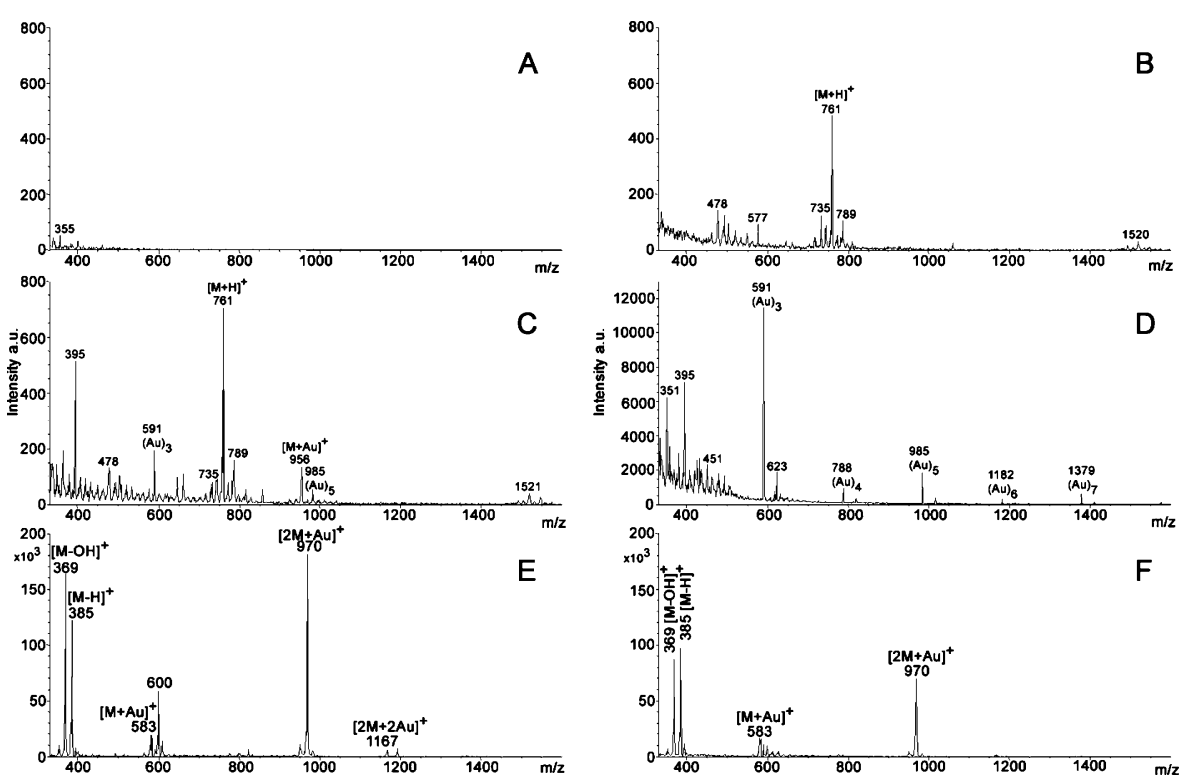

Figure 1. Influence of surface modification on PC and cholesterol signals in SIMS. (A) SIMS spectrum of PC. (B) ME-SIMS spectrum of PC in DHB (dried droplet). (C) SIMS spectrum of PC spotted on a gold-coated silicon wafer. (D) MetA-SIMS spectrum of PC spotted on a silicon wafer, covered by $1 \mathrm{~nm}$ of gold. (E) SIMS spectrum of cholesterol spotted on a gold-coated silicon wafer. (F) MetA-SIMS spectrum of cholesterol spotted on a silicon wafer, covered by $1 \mathrm{~nm}$ of gold.

$\mathrm{mg} / \mathrm{mL}$ solution of $\mathrm{DHB}$ in $50 \% \mathrm{MeOH} / 0.1 \%$ TFA was pumped for $10 \mathrm{~min}$ at a flow rate of $12 \mu \mathrm{L} / \mathrm{h}$ from a stainless steel capillary (o.d. $220 \mu \mathrm{m}$, i.d. $100 \mu \mathrm{m}$ ) held at $3.8 \mathrm{keV}$ and $5.0 \mathrm{~mm}$ over the grounded, maneuverable sample plate. Alternatively, a TLC sprayer (Sigma) was used to spray the same matrix solution for ME-SIMS, or $10 \mathrm{mg} / \mathrm{mL} \mathrm{HCCA} \mathrm{in} \mathrm{50 \%} \mathrm{EtOH/0.1 \%} \mathrm{TFA,} \mathrm{for}$ MALDI-MS. The nitrogen pressure required for efficient nebulization was $0.3-0.4$ bar.

Gold Deposition. Gold was sputter coated on the sample surface using a Quorum Technologies (Newhaven, East Sussex, U.K.) SC7640 sputter coater equipped with a FT7607 quartz crystal microbalance stage and a FT7690 film thickness monitor.

Mass Spectrometry. All static SIMS experiments were performed on a Physical Electronics (Eden Prairie, MN) TRIFTII time-of-flight SIMS (TOF-SIMS) equipped with an ${ }^{115} \mathrm{In}^{+}$liquid metal ion gun, as described before. ${ }^{46}$ Secondary ions were extracted through a $3.2-\mathrm{keV}$ electric field into the TOF analyzer and postaccelerated by an additional $8 \mathrm{keV}$ prior to detection on a dual multichannel plate/phosphor screen detector. In the MESIMS experiments, charge compensation was achieved by flooding the sample with low-energy electrons (29 eV, $40 \mathrm{nA}, 2-\mathrm{mm}$ diameter spot) between each primary ion pulse. To obtain images, the focused ion beam was randomly rastered across the sample. All experiments were performed with a primary ion beam current of $450 \mathrm{pA}$, a primary pulse length of $30 \mathrm{~ns}$, a spot diameter of 500 $\mathrm{nm}$, and a primary ion energy of $15 \mathrm{keV}$. The ion dose was such that all analyses were conducted in the static SIMS regime $(4.9$ $\times 10^{11}$ ions $/ \mathrm{cm}^{2}$ ). The instrument was calibrated on the low-mass fragments $\mathrm{CH}_{3}, \mathrm{C}_{2} \mathrm{H}_{5}$, and $\mathrm{C}_{3} \mathrm{H}_{5}$, which resulted in a mass accuracy for the imaging optimized instrument between 100 and 300 ppm, for the mass range between 0 and $1000 \mathrm{Da}$.

MALDI stigmatic imaging MS was performed on an extensively modified TRIFT-II instrument equipped with a nitrogen laser

(45) Hanton, S. D.; Cornelio Clark, P. A.; Owens, K. G. J. Am. Soc. Mass Spectrom. 1999, 10, 104-111.

(46) Schueler, B. W. Microsc. Microanal. Microstruct. 1992, 3, 119-139. source and a phosphor screen/CCD camera combination as described in detail by Luxembourg et al. ${ }^{47}$

MALDI Data Processing. Single-shot $200-\mu \mathrm{m}$ total ion count (TIC) MALDI stigmatic images were acquired and stored as tagged image file format (tiff) files with the corresponding synchronized ADC signals (Acqiris, Geneva, Switzerland). To image an entire rat brain, the sample stage was stepped at 100 $\mu \mathrm{m} / \mathrm{s}$ at a laser repetition rate of $9 \mathrm{~Hz}$ in a line scan. At the end of the line scan, the stage was stepped in the $y$ direction by 150 $\mu \mathrm{m}$. This process was repeated 62 times until the entire surface was imaged. Software was developed in-house to align all 1450 overlapping single-shot images into a line scan and, subsequently, all 63 line scans into an image. This high-resolution TIC image (resolving power $\sim 4 \mu \mathrm{m}$ ) was overlaid with the course resolution ADC data $(150 \times 11 \mu \mathrm{m})$ at each position, a color scale indicating the intensity of the selected $\mathrm{m} / \mathrm{z}$ for the image.

\section{RESULTS AND DISCUSSION}

SIMS, ME-SIMS, and MetA-SIMS of Lipids in DriedDroplet Samples. Figure 1A shows a SIMS spectrum of a total brain PC extract obtained with a $15-\mathrm{keV}^{115}$ In primary ion beam. Besides the low-mass fragments (not shown to prevent domination of the spectra), including the abundant choline headgroup fragment at $m / z 184$, no high-mass species are obtained. In Figure $1 \mathrm{~B}$, the PC extract is mixed with the MALDI matrix DHB and the ME-SIMS spectrum clearly shows the protonated molecular ions between $m / z 700$ and 800 , as well as the protonated lipid dimers around $m / z 1500$. In Figure $1 C$, a spectrum is shown of PC spotted on a gold-coated (1 nm) silicon wafer, measured with the same $15-\mathrm{keV}{ }^{115} \mathrm{In}$ primary ion beam. The spectrum is very similar to the one obtained with ME-SIMS. The only difference between the two spectra is the presence of a gold cationized species at $m / z 956$ in the spectrum of PC from the gold-coated target. However, the protonated species at $m / z 761$ shows a $\sim 5$ -

(47) Luxembourg, S. L.; Mize, T. H.; McDonnell, L. A.; Heeren, R. M. A. Anal. Chem. 2004, 76, 5339-5344. 

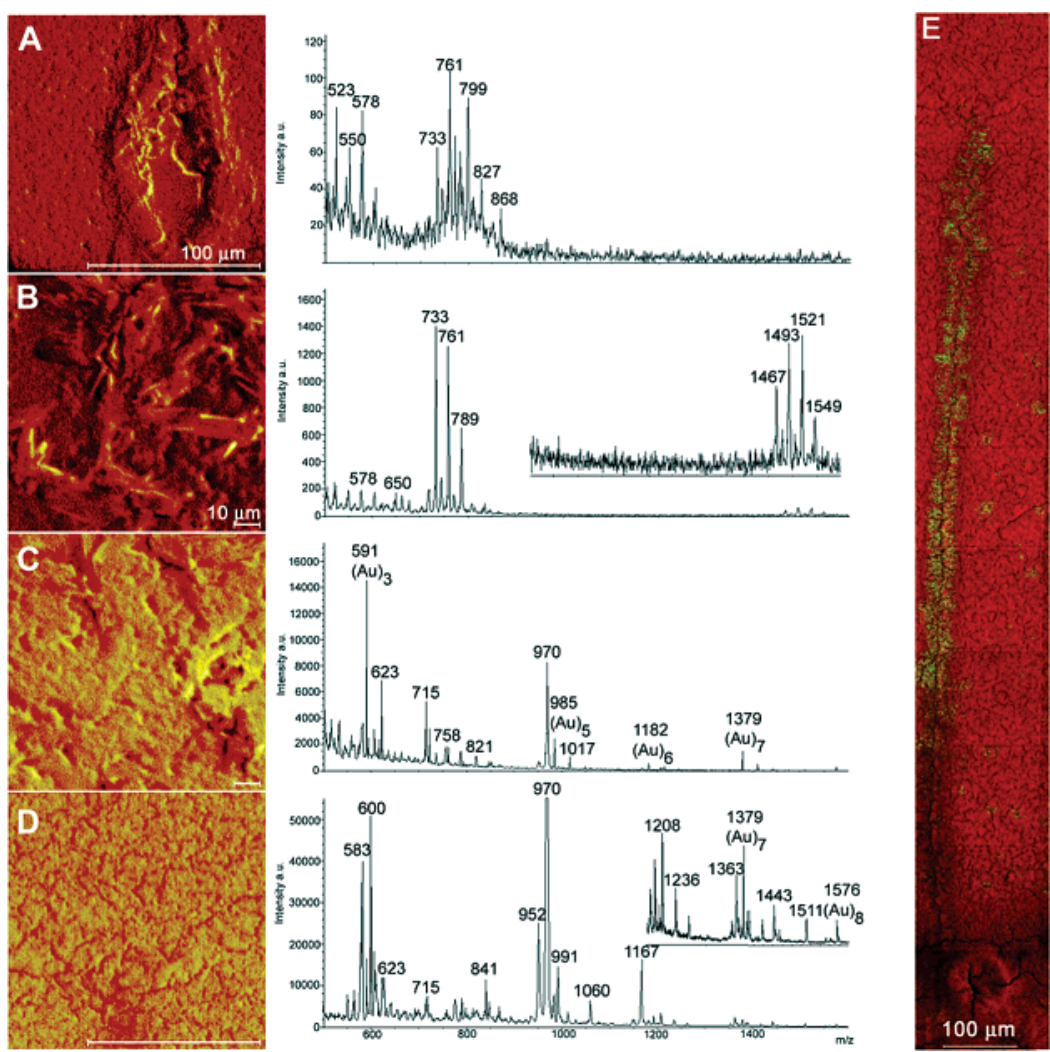

Figure 2. TIC images and corresponding mass spectra of rat brain tissue sections after surface modification. (A) SIMS analysis of an untreated tissue section (scale bar $100 \mu \mathrm{m}, 0-200$ counts). (B) ME-SIMS analysis of a tissue section after TLC sprayed DHB matrix (scale bar $10 \mu \mathrm{m}$, 0-350 counts). (C) ME-gold SIMS analysis of tissue sprayed with DHB and subsequently coated with 1-nm gold (scale bar 10 $\mu \mathrm{m}, 0-500$ counts). (D) MetA-SIMS analysis of a tissue section coated with 1-nm gold (scale bar $100 \mu \mathrm{m}, 0-1000$ counts). (E) Mosaic of 20 MetA-SIMS experiments of the ventricle area of a rat brain tissue section after deposition of $1 \mathrm{~nm}$ of gold with in red TIC (0-800 counts) and in green localization of the ion at $\mathrm{m} / \mathrm{z} 1443(0-3$ counts) (scale bar $100 \mu \mathrm{m})$. The total ion dose was $1.8 \times 10^{12}$ ions $/ \mathrm{cm}^{2}$ in panels A, D and E, $5.1 \times$ $10^{12}$ ions $/ \mathrm{cm}^{2}$ in panel $\mathrm{B}$, and $1.97 \times 10^{12} \mathrm{ions} / \mathrm{cm}^{2}$ in panel $\mathrm{C}$.

fold higher intensity over the cationized PC. The same behavior is observed for other lipid species deposited on a gold-coated sample target. Since most of the PC will be present on the sample in its protonated form, the intensity enhancement effect of the gold-coated target seems to be more related to enhanced lipid desorption/ionization than cationization. Figure 1D shows an experiment where first the $\mathrm{PC}$ is spotted on a silicon wafer after which $1 \mathrm{~nm}$ of gold is deposited on top of the sample. The sample was measured directly after metal coating, after 3 and $6 \mathrm{~h}$, and the following day. The spectrum shows intense gold cluster ions, but the protonated or cationized molecular ion species of PC could not be detected. Another PC sample was placed in an oven, heated to $60{ }^{\circ} \mathrm{C}$, and measured at similar time points, but here also no $\mathrm{PC}$ ions could be found. These results are in contrast to polymer studies, where a 2-nm overlayer of gold yielded either intense metal cationized species or signal enhancement for the protonated species. ${ }^{28} \mathrm{~A}$ study by Adriaensen et al..$^{31}$ showed that the molecular information became visible after one or two weeks waiting, but this approach is biologically irrelevant. For comparison, the spectra in Figure 1E and F show the SIMS spectra of cholesterol where either the cholesterol is spotted on the gold-coated target (E) or the gold is sputtered on top of the cholesterol $(\mathrm{F})$. In both cases, abundant cholesterol-derived pseudomolecular ions can be seen at $m / z 369[\mathrm{M}-\mathrm{OH}]^{+}$and $m / z 385[\mathrm{M}-\mathrm{H}]^{+}$as well as cationized species at $m / z 583[\mathrm{M}+\mathrm{Au}]^{+}, m / z 970[2 \mathrm{M}+\mathrm{Au}]^{+}$, and $m / z 1167[2 \mathrm{M}+2 \mathrm{Au}]^{+}$. The cholesterol sample gave the abundant signals right after metal coating. These results point to a molecule specific enhancement of the gold overlayer.

SIMS, ME-SIMS, and MetA-SIMS in Direct Imaging of Rat Brain Tissue Sections. Raising the complexity of the biomolecular surface from a moderately complex PC mixture to a brain tissue section poses a challenge for ME-SIMS and MetA-SIMS (or any other MS imaging technique). The $10-\mu \mathrm{m}$-thick rat brain cryosections were cut at the level of the hypothalamus, using a cryomicrotome. Figure $2 \mathrm{~A}$ shows a SIMS experiment directly on the tissue surface. In the spectrum, different diacylglycerol (DAG) and phospholipid species can be seen, however, at relatively low signal intensities. In the SIMS-only experiment, the TIC image shows a high-resolution image of the ventricle but the phospholipid signal intensities are too low to allow molecule-specific imaging experiments. To enhance molecular signal intensities in a previous study, we used electrospray matrix deposition yielding micrometer-sized matrix crystals at the tissue section surface. ${ }^{24}$ In our current study, we use a TLC sprayer resulting in matrix crystal sizes of $\sim 20 \mu \mathrm{m}$. To improve the crystallization of these relatively large crystals, the tissue was washed in ice-cold $70 \%$ ethanol solution. These large matrix crystals result in a significant increase of the phospholipid signals shown in Figure 2B. The TIC image, however, is completely dominated by matrix crystal structures and the phospholipid signals colocalize within these crystal structures. This example clearly shows that matrix deposition resulting in large matrix crystals compromises the visible 
detail in SIMS analysis. Comparing panels A and B in Figure 2, an increase in signal intensity by 1 order of magnitude is observed for PC while the ceramide and DAG signals in the $m / z 500-600$ range increase only by a factor of 2 , indicating reduced fragmentation in ME-SIMS. Except for the lipid signals, the spectrum shows no other molecular signals in ME-SIMS, while measuring this same section in our stigmatic MALDI instrument yielded signals up to $4000 \mathrm{Da}$. The major difference between the two approaches relates to the amount of sample consumed. While in SIMS the penatration depth is typically $10 \mathrm{~nm}$, in MALDI each laser shot consumes approximately $100 \mathrm{~nm}-1 \mu \mathrm{m}$ of sample. A study by Hanton et al. ${ }^{45}$ showed that segregation of different molecular species occurs during crystallization of the matrix, and that MESIMS and MALDI yield different spectra for (complex) mixtures. These results agree with our observations where ME-SIMS and MALDI analysis of the same tissue section yield markedly different spectra. During crystallization, the surface-active lipids largely migrate to the crystal surface where they are readily accessible to the SIMS primary ion beam. Conversely, salts are excluded from the crystals altogether while peptides are mainly incorporated into the matrix crystals, the latter resulting in intense MALDI signals. To image peptide distributions with ME-SIMS, the crystal sizes have to be in the order of the sampling depth of the primary ion beam, strongly compromising sensitivity.

Figure $2 \mathrm{C}$ shows the effect of $1 \mathrm{~nm}$ of gold on top of the matrix crystals on the tissue. This approach results in reduced surface charging and therefore preserves the ion-optical image quality in stigmatic MALDI imaging. Furthermore, gold coating has been shown to increase signal intensity in conventional microprobe MALDI imaging. ${ }^{48}$ In Figure $2 \mathrm{C}$, the dominant phospholipid signals have largely disappeared, and in addition to gold cluster signals, multiple novel signals are observed, which cannot be assigned to any of the commonly observed lipids. Inspecting the localization of the peaks observed in the gold-covered ME-SIMS spectrum reveals that several of the gold-enhanced ions localize within the crystals (for example $m / z 970$; data not shown), while others are present over the entire imaged area. Figure 2D shows the spectrum of a MetA-SIMS experiment, where a 1-nm layer of gold was deposited on an unwashed tissue section. Several of the observed peaks correspond to those in the gold-coated ME-SIMS spectrum, including the very abundant cholesterol $[2 \mathrm{M}+\mathrm{Au}]^{+}$ peak at $m / z 970$. Several of the other abundant peaks (i.e., $m / z$ $583,600,970,952$, and 1167) can also be attributed to cholesterol as can be seen from a comparison with Figure 1E. In the higher mass range, however, species are observed that do not correspond to cholesterol or the cationized lipid species, which are normally observed in SIMS spectra. These peaks remain yet unassigned, illustrating the need for SIMS tandem MS. That these species are tissue derived and not introduced by the gold coating can be seen from the overlay of the ion at $m / z 1443$ (green) with the TIC image of the ventricle area in the rat brain (red), depicted in Figure 2E. Here, a rat brain tissue section was covered with 1 nm of gold and the sample stage was stepped in a mosaic pattern, after which the resulting images were aligned to produce a single molecular image of the ventricle area in the rat brain. As can be seen from this image, the ion at $m / z 1443$ localizes very precisely

(48) Stoeckli, M.; Schweitzer, A.; Knochenmuss, R.; McCombie, G.; Staab, D.; Rohner, T., 53rd ASMS Conference, San Antonio, TX, 2005. within the ventricle as well as in some select areas in the neighboring tissue. Furthermore, these results show that the use of gold produces images with very high spatial resolution. In addition to the newly observed ions, it is clear from inspection of the intensity axes in Figure 2A-D that progressively the absolute signal intensity increases over 2 orders of magnitude. These high count rates are essential for imaging experiments where image contrast largely relies on the dynamic range within an image (also see Figures 3 and 6).

ME-SIMS Imaging of Single Neuroblastoma Cells. In tissue sections, the majority of the cells are transversely cut by the cryomicrotome displaying both membrane ridges between adjacent cells as well as cytosolic components and organelles. Single cells, however, grown on glass slides, frozen, and dried, pose a fundamentally different type of biological surface. In single-cell imaging MS studies, mainly cell surface components are likely to be sampled by the primary ion beam. Since ME-SIMS and especially MetA-SIMS are capable of imaging biological surfaces with very high spatial resolution, these techniques could be very well suited for direct analysis of lipid distributions on single-cell surfaces. For ME-SIMS the cells, grown on conductive ITO-coated glass slides, were covered with a thin layer of matrix by electrospray deposition yielding small $(0.3-1 \mu \mathrm{m})$ matrix crystals. The crystal sizes obtained with the TLC sprayer, shown in Figure $2 \mathrm{~B}$ and $\mathrm{C}$, were in the order of $20 \mu \mathrm{m}$. Since a typical neuroblastoma cell is around $50 \mu \mathrm{m}$, one such crystal would cover half the cell body and thus the morphology of the matrix crystals, instead of the cell, would dominate the MS image. A second reason for the use of electrospray deposition is the already mentioned thickness of the matrix layer and the SIMS sampling depth.

Figure 3 shows the capability of ME-SIMS to image the molecular ion distribution of intact lipids directly from single neuroblastoma cells. The ME-SIMS spectrum of these cell surfaces shows several lipid species in the mass range between $m / z 700$ and 900, ceramide and DAG species between $m / z 500$ and 700, and organic fragments in the lower mass range. The phospholipids; phosphatidylcholine and sphingomyelin (SM) can be readily identified (Table 1), together with the pseudomolecular ion signal for cholesterol at $m / z 385[\mathrm{M}-\mathrm{H}]^{+}$and the dominant fragment at $m / z 369[\mathrm{M}-\mathrm{OH}]^{+}$. For comparison, using conventional SIMS without any matrix, only elements and lowmass organic fragments such as the PC headgroup $(m / z 184)$ are observed.

Panels A and B in Figure 3 show ME-SIMS images of the distribution of cholesterol in neuroblastoma cells. The images show the distribution of the dominant cholesterol fragment ion at $m / z 369$, over a $75 \times 75 \mu \mathrm{m}$ area. Figure 3A clearly shows three separate cells present in the imaged area, with the bottom cell only partly sampled by the SIMS ionization beam. The images show high cholesterol signal intensity at the border of the cells, the plasma membrane, compared to the center of the cells, corresponding to the nuclei. This observation is in line with previous observations where cholesterol was found primarily in the plasma membrane. ${ }^{24,25,49}$

Panels $\mathrm{C}-\mathrm{F}$ in Figure 3 show ME-SIMS images of the same single neuroblastoma cell. The images correspond to a field of

(49) Sjovall, P.; Lausmaa, J.; Nygren, H.; Carlsson, L.; Malmberg, P. Anal. Chem. 2003, 75, 3429-3434 


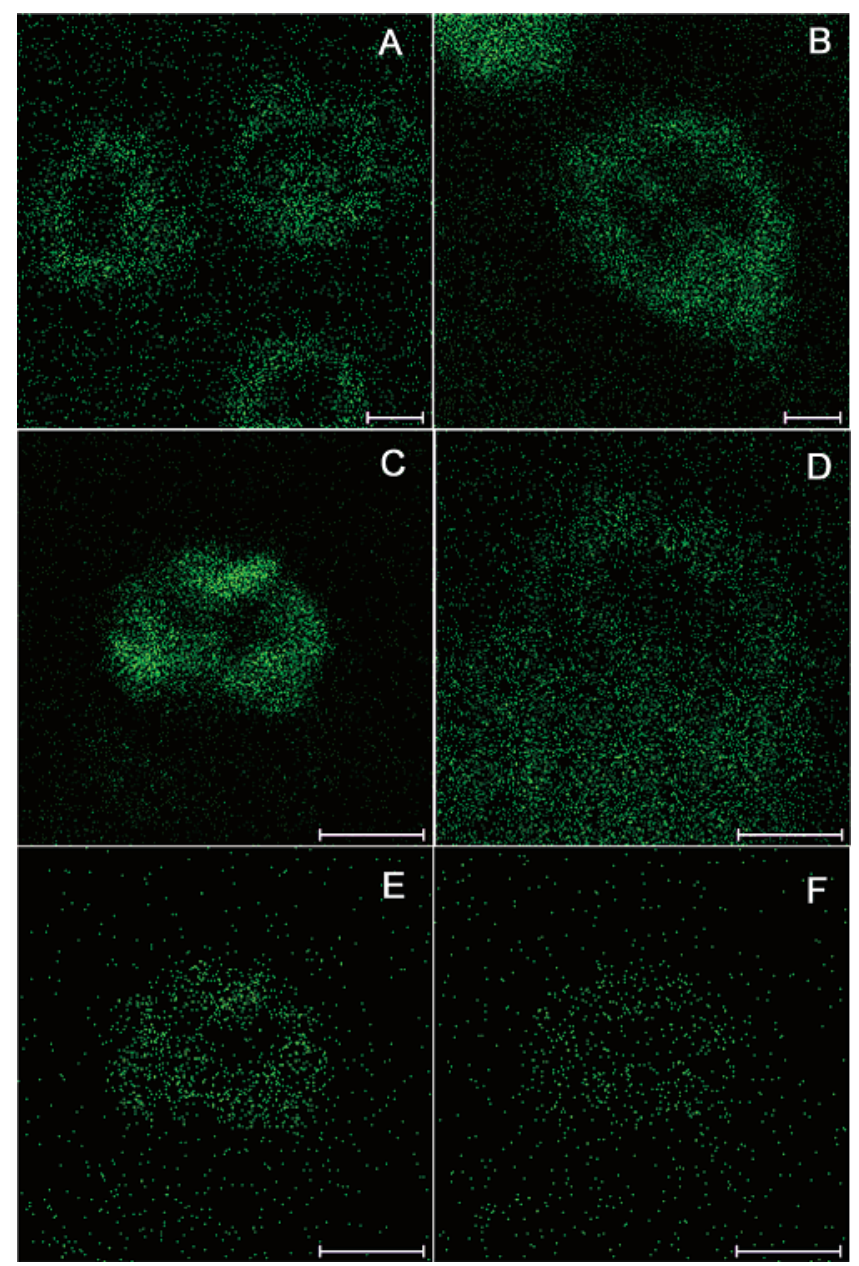

Figure 3. Molecular images of single neuroblastoma cells obtained with ME-SIMS. SIC images are (A) cholesterol fragment $[\mathrm{M}-\mathrm{OH}]^{+}$ $(\mathrm{m} / \mathrm{z} 369,0-5$ counts), (B) cholesterol fragment $(\mathrm{m} / \mathrm{z} 369,0-7$ counts), (C) PC headgroup ( $\mathrm{m} / \mathrm{z} 184,0-7$ counts), (D) cholesterol fragment ( $m / z$ 369, $0-4$ counts), (E) PC ( $m / z 761,0-3$ counts), and (F) SM ( $\mathrm{m} / \mathrm{z} 787,0-3$ counts). Panels $\mathrm{C}-\mathrm{F}$ are acquired in the same experiment from the same cell. Scale bar in all images, $10 \mu \mathrm{m}$. Total ion dose $(A, B) 2.8 \times 10^{12}$ and $(C-F) 8.0 \times 10^{12} \mathrm{ions} / \mathrm{cm}^{2}$.

\section{Table 1. Phosphatidylcholine and Sphingomyelin Signals Observed in Single-Cell ME-SIMS Imaging Experiments}

$m / z$

733

747

759

761

783

787

789

809

811 ion

$$
\begin{aligned}
& \left.[\mathrm{SM}+\mathrm{H}]^{+} \text {18:0/16:1/[PC }+\mathrm{H}\right]^{+} 16: 0 / 16: 1 \\
& {[\mathrm{PE}+\mathrm{H}]^{+} \text {18:0/18:1 }} \\
& \left.[\mathrm{SM}+\mathrm{H}]^{+} \text {20:1/16:1/[PC }+\mathrm{H}\right]^{+} 16: 0 / 18: 2 \\
& {[\mathrm{PC}+\mathrm{H}]^{+} \text {16:0/18:1 }} \\
& {[\mathrm{PC}+\mathrm{Na}]^{+} 16: 0 / 18: 1} \\
& {[\mathrm{SM}+\mathrm{H}]^{+} 22: 0 / 16: 1 /[\mathrm{PC}+\mathrm{H}]^{+} 18: 1 / 18: 1} \\
& {[\mathrm{PC}+\mathrm{H}]^{+} 18: 0 / 18: 1} \\
& {[\mathrm{PC}+\mathrm{H}]^{+} 20: 4 / 18: 1} \\
& {[\mathrm{PC}+\mathrm{H}]^{+} 20: 3 / 18: 1}
\end{aligned}
$$

view of $40 \times 40 \mu \mathrm{m}$ and are centered on the $m / z 184$ signal, which is associated with the nuclear membrane. ${ }^{49}$ The signals corresponding to the PC headgroup (Figure 3C) and the molecular ions of $\mathrm{PC}$ and SM, panels $\mathrm{E}$ and $\mathrm{F}$, respectively, are all localized in the nuclear region. The cholesterol signal, and thus the plasma membrane, is partially outside the field of view. The lipid species are predominantly present along the border of the nucleus, as

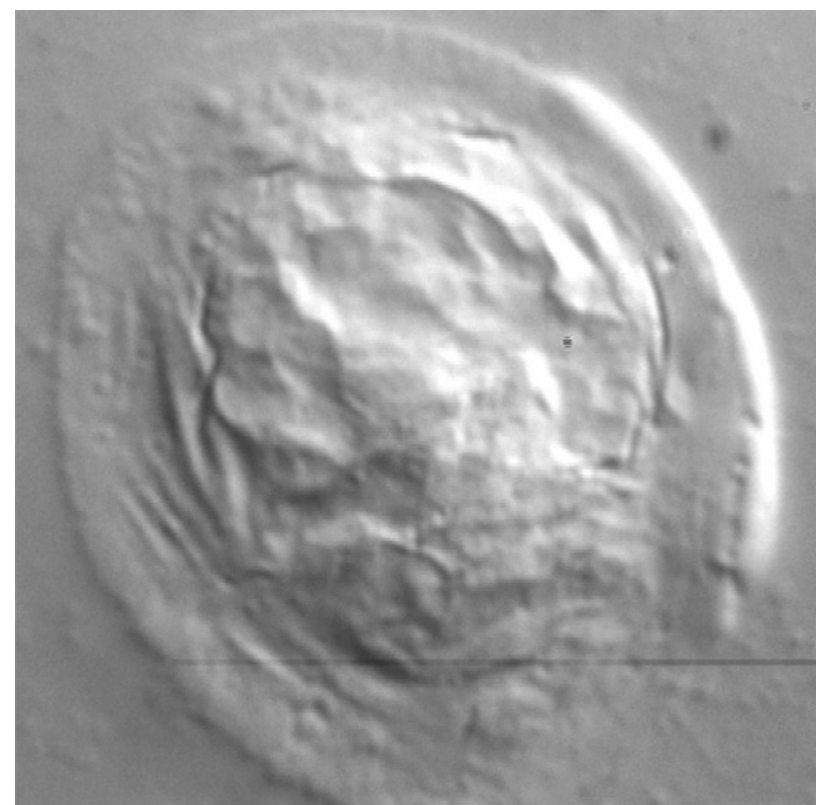

Figure 4. DIC microscope image of a single neuroblastoma cell after washing and drying. Field of view, $50 \times 50 \mu \mathrm{m}$.

building blocks of the nuclear membrane. The nuclear signals are surrounded by the cholesterol signal, corresponding to cellular outline formed by the plasma membrane (Figure 3D). The signal of ceramide species colocalize with the cholesterol signal (data not shown), in line with several reports. ${ }^{50-53}$ Nuclear membrane components dissolve in the matrix solution upon spraying. This can occur when the plasma and nuclear membranes make contact during the drying process. Figure 4 shows an optical image (50 $\times 50 \mu \mathrm{m})$ of a single neuroblastoma cell obtained with a differential interference contrast (DIC) microscope. The image clearly shows that the outside rim of the cell is much flatter than the center where the nucleus is located. To keep the damage to the cells as limited as possible, the cells are first washed in a $300 \mathrm{mM}$ sucrose solution to wash away the salts from the growth medium while preventing hypotonic shock resulting in cell lysis. For this reason the second wash step, with Milli-Q water, is a quick rinse followed by prompt drying in a vacuum chamber. The timing of the Milli- $Q$ wash is critical; exposure of the cells to water longer than $30 \mathrm{~s}$ results in hypotonic shock for $>90 \%$ of the cells. Although intact lipids such as PC and SM are localized within the cellular outlines by ME-SIMS, a higher resolution MS technique in combination with a higher dynamic range and higher intensity signals would improve SIMS for molecular imaging of complex biological surfaces. Gold coating, analogous to tissue imaging (Figure 2D), can be used to enhance the ion yields from single cells.

MetA-SIMS Imaging of Single Neuroblastoma Cells. In Figure 5, the MetA-SIMS spectrum of single neuroblastoma cell surfaces coated with 1-nm gold is shown. In panel A, the lowest mass range $(m / z 0-250)$ was blanked to prevent saturation of the detector with low-mass ions, with no further effect on the

(50) Andrieu, N.; Salvayre, R.; Levade, T. Eur. J. Biochem. 1996, 236, 738-745.

(51) Birbes, H.; El Bawab, S.; Hannun, Y. A.; Obeid, L. M. FASEB J. 2001, 15, 2669-2679.

(52) Liu, P. S.; Anderson, R. G. W. J. Biol. Chem. 1995, 270, 27179-27185.

(53) Grassme, H.; Jendrossek, V.; Riehle, A.; von Kurthy, G.; Berger, J.; Schwarz, H.; Weller, M.; Kolesnick, R.; Gulbins, E. Nat. Med. 2003, 9, 322-330. 


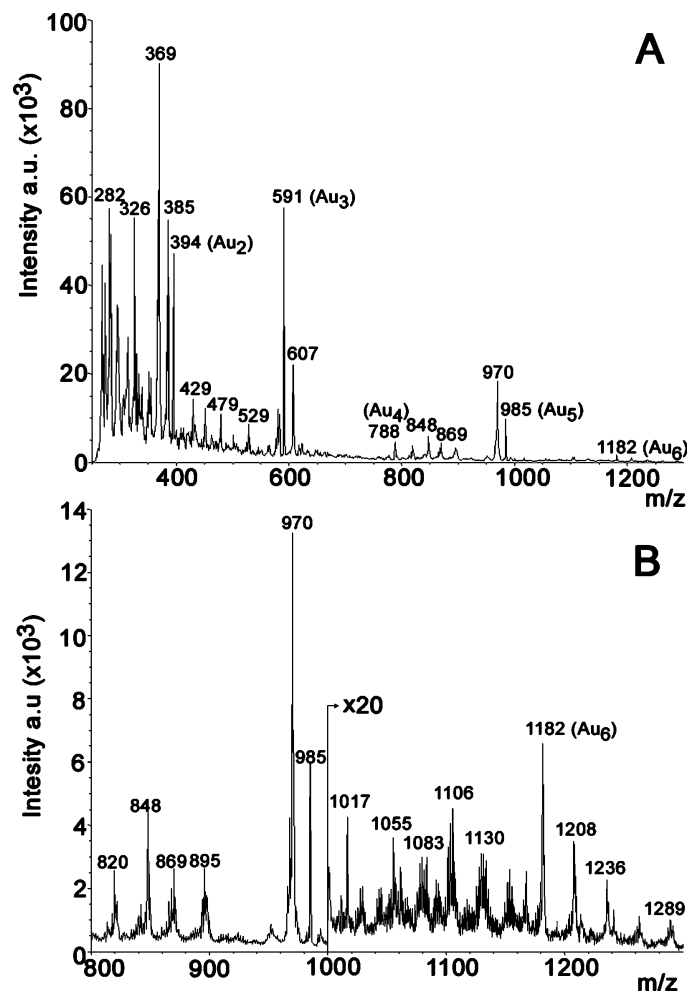

Figure 5. MetA SIMS spectrum of neuroblastoma cells after deposition of 1-nm gold. (A) Mass range 250-1300 Da; low-mass ions ( $<250 \mathrm{Da}$ ) were blanked to prevent detector saturation. (B) The same spectrum, zoomed-in on the 800-1300-Da mass range. The spectrum was obtained by sampling a $150 \times 150 \mu \mathrm{m}$ area by the primary ion beam. $(\mathrm{Au})_{n}$ marks indicate the gold cluster $\mathrm{m} / \mathrm{z}$ values.

measurement. In the mass range between $m / z 250$ and 500, a very abundant pseudomolecular ion signal of cholesterol $(\mathrm{m} / z 385)$ together with the dominant fragment ion $(\mathrm{m} / z$ 369) can be observed. Upon gold deposition, a very rich mass spectral signature is observed consisting of signals in the $\mathrm{m} / z$ 500-1300 range. No readily interpretable masses were observed other than cholesterol and a DAG species $(m / z 607)$. Count rates for most ions in the spectrum were high enough to allow imaging and thus localization of the signals. Almost all of the species observed, other than the gold clusters, colocalize with the neuroblastoma cell surfaces. The use of gold to enhance the secondary ion yield also greatly enhances the image quality in imaging SIMS experiments, ${ }^{29}$ as can be seen in Figure 6. Figure 6 shows selected ion count (SIC) images of three separate experiments (panels A and $\mathrm{B}, \mathrm{C}$ and $\mathrm{D}$, and panel $\mathrm{E})$. The first four rows (A-D) show images of multiple cells within a $150 \times 150 \mu \mathrm{m}$ field of view. The last row (E) shows an image of a single cell in a $75 \times 75 \mu \mathrm{m}$ field of view. Several localization patterns can be distinguished: in panel A, cholesterol is found on the entire cell surface, with higher intensity at the cellular outlines, whereas the $m / z 607$ signal is highly intensive at the outline only. In panel $\mathrm{B}$, the localization of the pseudomolecular cholesterol ion at $m / z 970$ follows that of the $m / z 369$ pseudomolecular ion; however, the increased signal intensity at the cell outlines is not observed. This difference in localization between different pseudomolecular ions of the same molecular species had been observed before and is described elsewhere..$^{54}$ The $m / z 1080$ signal in panel B shows a distribution throughout the cell surface and only the overlay of $m / z 970 / 1080$

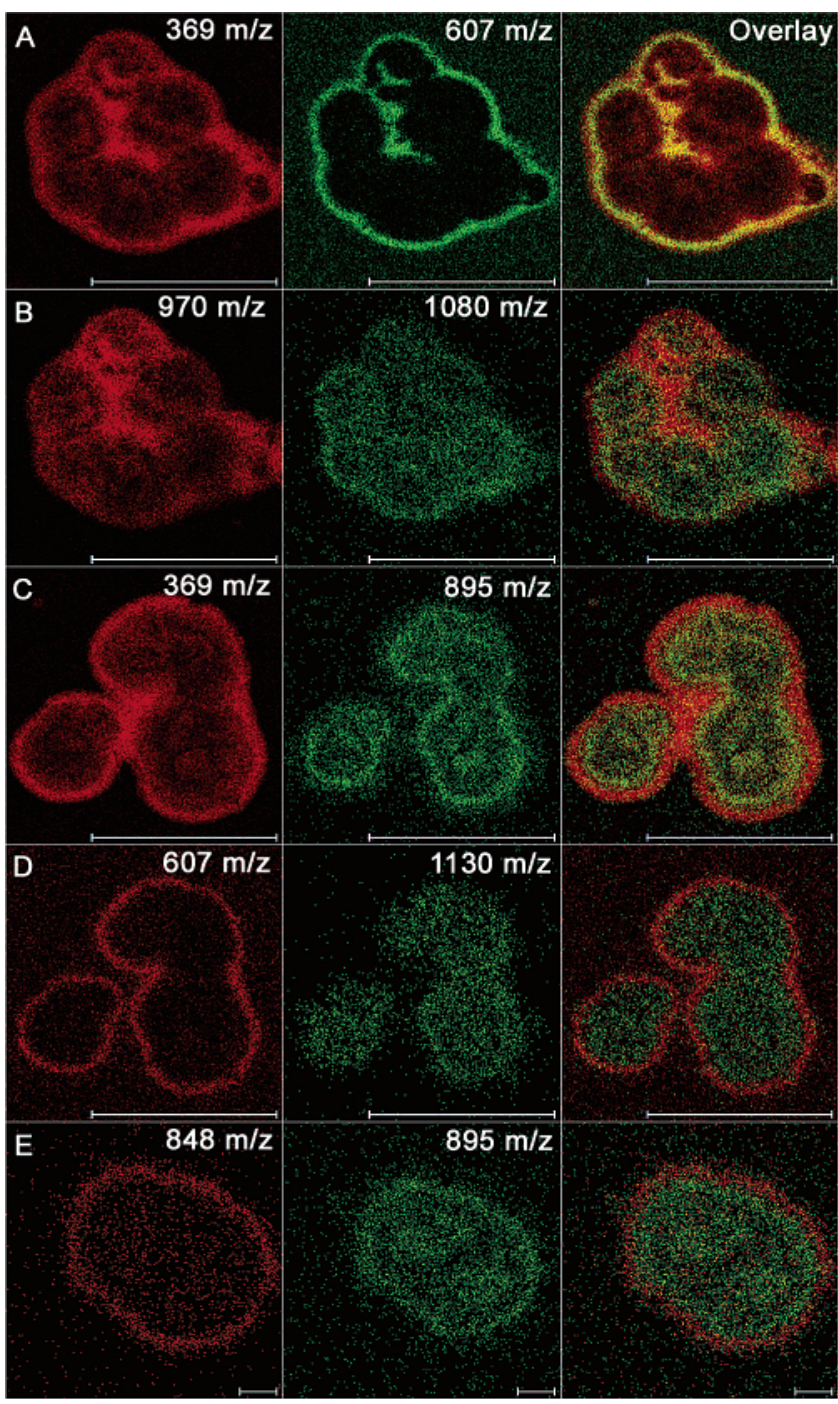

Figure 6. Cellular localization of MetA-SIMS selected ion count signals from neuroblastoma cells. Cells were imaged after deposition of 1-nm gold. (A) $\mathrm{m} / \mathrm{z} 369$ (cholesterol $[\mathrm{M}-\mathrm{OH}]^{+}, 0-14$ counts) and 607 (DAG, 0-6 counts). (B) $\mathrm{m} / z 970$ (cholesterol [2M + Au] $]^{+}, 0-4$ counts) and $1080\left(0-1\right.$ counts). (C) $\mathrm{m} / z 369$ (cholesterol $[\mathrm{M}-\mathrm{OH}]^{+}$, 0-12 counts) and 895 (0-2 counts). (D) $\mathrm{m} / z 607$ (DAG, $0-4$ counts) and 1130 (0-1 counts). (E) $\mathrm{m} / \mathrm{z} 848$ (0-1 counts) and $895(0-1$ counts). $(\mathrm{Au})_{n}$ marks indicate the gold cluster $\mathrm{m} / \mathrm{z}$ values. Scale bar: $100 \mu \mathrm{m}$ in panels $\mathrm{A}-\mathrm{D}$ and $10 \mu \mathrm{m}$ in panel $\mathrm{E}$. Total ion dose $1.18 \times$ $10^{12}$ ions $/ \mathrm{cm}^{2}$ in panels $A-D$ and $4.7 \times 10^{12}$ ions $/ \mathrm{cm}^{2}$ in panel $\mathrm{E}$.

shows that at the cellular outlines the $m / z 1080$ signal is absent, illustrating the power of overlaying SIC images in two colors to pinpoint subtleties in localization. In panel C, the cholesterol and $m / z 895$ distributions show an intermediate pattern, between A and B. The $m / z 895$ signal localizes at the cellular surface with increased intensity at the outlines and on first sight colocalizes with cholesterol. However, the overlay shows that the cellular outline is highlighted by the cholesterol signal whereas $m / z 895$ localizes on the cell surface, like $m / z 1080$. Similar behavior is observed for $m / z 607$ and 1130 in panel $\mathrm{D}$, which shows the same cell cluster. Also, in panel $\mathrm{E}$, this type of localization is observed for $m / z 848$ and 895. Although identification of the well-localized

(54) Altelaar, A. F. M.; van Minnen, J.; Heeren, R. M. A.; Piersma, S. R. Appl. Surf. Sci., accepted. 

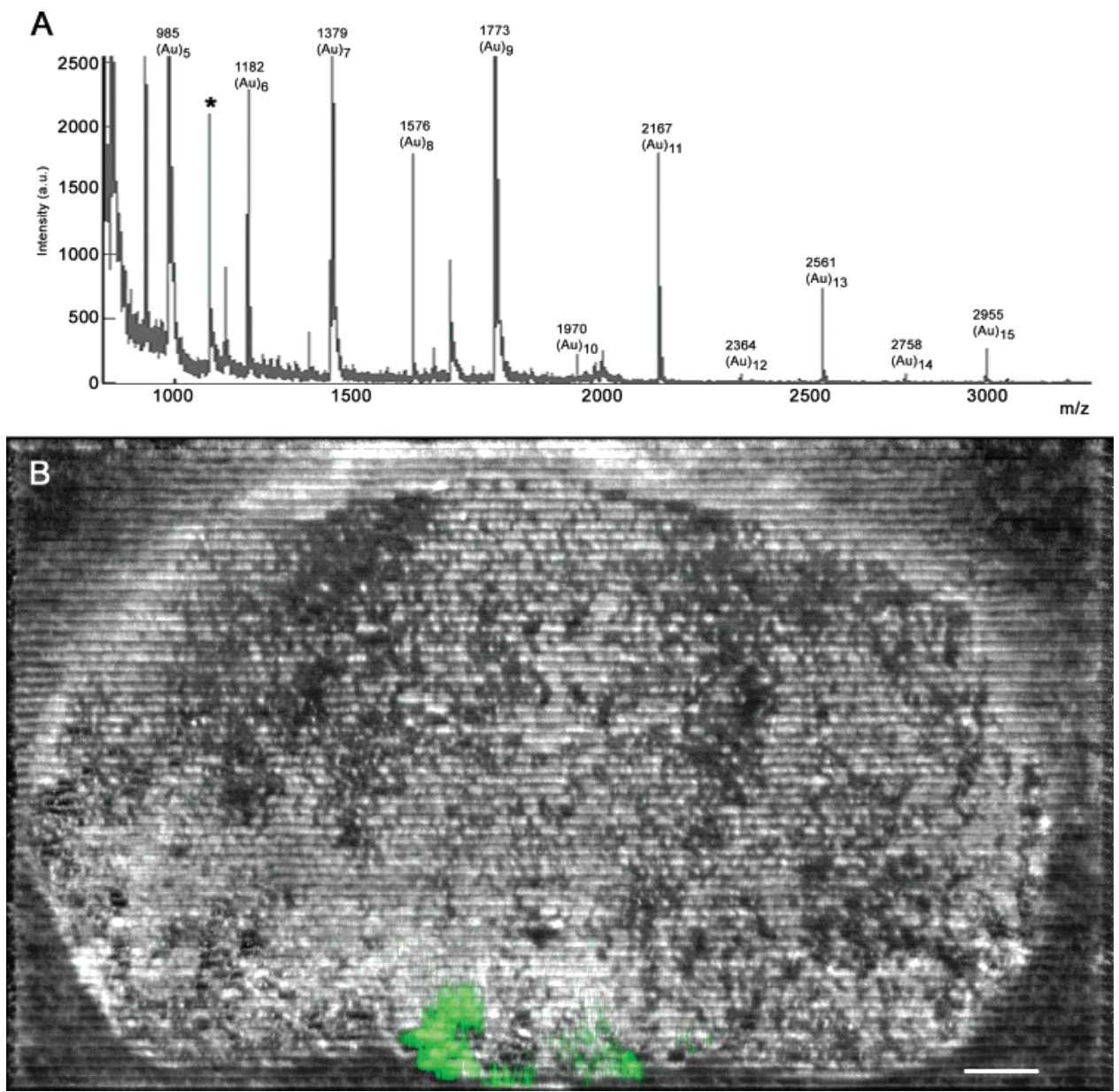

Figure 7. MALDI stigmatic imaging of a rat brain tissue section. (A) A line scan summed mass spectrum showing gold cluster peaks and several peptide peaks, with the vasopressin mass at $\mathrm{m} / \mathrm{z} 1085{ }^{*}$ ). (B) TIC image (gray scale) of a HCCA-coated rat brain tissue section overlaid with the selected ion image for vasopressin (green). Scale bar, $1 \mathrm{~mm}$.

signals in Figure 6 is required for the technique to be relevant for biology, the potential of MetA-SIMS for biological surface imaging is high. Compared to ME-SIMS, the lateral resolution of MetA-SIMS is higher. In our previous ME-SIMS imaging study of Lymnaea stagnalis nervous tissue, ${ }^{24}$ we calculated an experimental resolving power. The resolving power is a convolution of the SIMS instrumental spatial resolution and the sample handling/ matrix deposition protocol. The resolving power is calculated from a line scan analysis. For the cholesterol image, shown in Figure $6 \mathrm{~A}$, the MetA-SIMS resolving power is $1.5 \mu \mathrm{m}$, a factor of $\sim 2$ better than determined previously for ME-SIMS. ${ }^{24}$ In Figure $6 \mathrm{C}$, one can readily distinguish subcellular details in both the cholesterol and the $m / z 895$ distributions.

Comparison of SIMS, ME-SIMS, and MetA-SIMS for Single-Cell Imaging. Using conventional static SIMS, several lipids are observed but no intact-molecule imaging is possible at the cellular level. However, the technique does not require any surface modification and therefore does not introduce artifacts. In a ME-SIMS spectrum, several lipid species and their fragments can be identified and imaged but signal intensities in the higher mass range are still relatively low. To increase the yield of the higher mass species, larger matrix crystals are required, compromising spatial detail. Furthermore, ME-SIMS requires extensive surface modification since surface analytes have to be incorporated in the matrix crystals (i.e., surface wetting will occur).
Conversely, MetA-SIMS relies on a gas-phase deposition process and, therefore, does not require liquid-surface interaction. In MetA-SIMS, lateral analyte diffusion is minimized, only on the nanmeter scale migration on gold islands may occur. The signal intensity of cholesterol in the MetA-SIMS experiment is $\sim 50$ times higher compared to the DHB-coated samples. In MetA-SIMS, the area where abundant phospholipid peaks could be seen in the ME-SIMS spectrum has changed dramatically. In the region between $m / z 500$ and 800 , where in the ME-SIMS spectrum ion signals corresponding to ceramide, DAG, and phospholipid species could be found, almost no signal can be seen. The region between $m / z 800$ and 1300 on the other hand shows a large number of peaks, which are not obtained by ME-SIMS. The MetA-SIMS spectra in Figures 2 and 5 are quite similar, indicating that MetASIMS signals are most likely generic cell membrane components such as lipids. For MetA-SIMS to become a biological relevant technique, these peaks have to be assigned, again showing the need for tandem MS in SIMS.

The mechanism of MetA-SIMS and the role of the gold layer are still unclear. Previously, migration of mobile analytes onto gold nanoislands has been proposed. ${ }^{27,38}$ Atomic force microscopy (AFM) showed that gold islands in the order of $100 \mathrm{~nm}$ are formed on tissue (data not shown). Indeed, increased signal intensities were obtained for a mobile analyte like cholesterol when gold was deposited on a thin cholesterol layer. However, migration of 
surface-active phospholipids onto gold nanoislands is not seen, not from the thin-layer preparation or from the tissue sections or cells. Delcorte et al. ${ }^{27}$ already showed this explanation to be questionable for larger molecules (2000 Da). Another explanation is the increased stopping power by a layer of gold. However, stopping power is only increased if the sample surface is gold and the analytes are deposited as a thin (mono-)layer on top, this is not the case for tissues or cells covered by gold. Yet another mechanism includes gold as cationizing agent leading to improved ion formation. ${ }^{28,40}$ Indeed, several of the observed signals are cationized by gold in the MetA-SIMS experiments. However, the dominant species found in SIMS and ME-SIMS analysis are not found in MetA-SIMS, neither protonated nor cationized. Furthermore, gold deposition on the matrix crystals in the ME-SIMS analysis, revealed species within these crystals not observed before. These observations point to a molecule-selective enhancement by the sputtered gold.

Gold-Enhanced MALDI Imaging of Rat Brain Sections. Not only for SIMS but also for MALDI MS imaging, gold deposition results in enhanced image quality and signal intensity. In conventional microprobe MALDI imaging MS $(\sim 100-\mu \mathrm{m}$ laser spot) gold deposition on matrix-covered tissue sections resulted in enhanced signal intensity. ${ }^{48}$ Here we show the effect of gold deposition on tissue using a stigmatic imaging MALDI mass spectrometer. In stigmatic imaging, a large surface area $(200 \mu \mathrm{m})$ is illuminated by a laser and the spatial origin of the desorbed molecules is retained during the time-of-flight separation. ${ }^{47}$ In stigmatic MALDI imaging, the MS image quality is not related to laser spot size but is related to the quality of the ion optics. The stigmatic TRIFT II instrument ion optics are sensitive to changes in the sample potential. Isolating samples such as tissue sections are prone to charging (and hence potential bias) and therefore result in extensively blurred stigmatic images. Deposition of 5 nm of gold on top of pneumatically sprayed HCCA matrix reduces charging of the tissue section and, therefore, improves stigmatic MALDI image quality. In Figure 7, a stigmatic MALDI imaging experiment of rat brain tissue is shown. To image a large surface area, such as a rat brain tissue section, the sample stage is stepped under a continuously firing laser beam. Single-shot images and spectra are recorded at $9 \mathrm{~Hz}$ while the sample stage moves in a line scan at $100 \mu \mathrm{m} / \mathrm{s}$. In Figure 7A, the summed mass spectrum of a line scan over the rat brain is shown. Multiple well-resolved peaks in the 800-2500-Da range are obtained, among which is a dominant signal for the neuropeptide vasopressin at $m / z 1085\left(^{*}\right)$. Figure 7B shows 63 line scans, of 1450 individual images each, aligned using in-house-developed software (spatial image composer 0.8 ) to construct a rat brain TIC image (gray scale). The

(55) Wotjak, C. T.; Kubota, M.; Liebsch, G.; Montkowski, A.; Holsboer, F.; Neumann, I.; Landgraf, R. J. Neurosci. 1996, 16, 7725-7732.

(56) Ludwig, M. J. Neuroendocrinol. 1998, 10, 881-895.

(57) Hernando, F.; Schoots, O.; Lolait, S. J.; Burbach, J. P. H. Endocrinology 2001, 142, 1659-1668.
TIC image is overlaid with the molecule-specific image of vasopressin (green), which is constructed from the mass spectral data of the individual laser shots. Imaging MS localizes vasopressin in the hypothalamic area around the third ventricle, where it is known to be synthesized and released, ${ }^{55,56}$ and where the V1b vasopressin receptor has been localized previously by immunohistochemistry. ${ }^{57}$ The same experiment omitting gold deposition resulted in both blurred TIC images and reduced signal intensity (neuropeptide localization was not possible). The results shown in Figure 7 could be obtained only after gold deposition.

\section{CONCLUSIONS}

ME-SIMS enhances lipid molecular ion signals over conventional static SIMS at the expense of surface analyte diffusion and surface corrugation by matrix deposition. MetA-SIMS shows highly enhanced as well as new signals from cells and tissues after gold deposition without lateral analyte diffusion, but analyte identification is required for interpretation of the high-resolution ion images in a biological context.

The effect of gold deposition on MALDI stigmatic imaging is most likely related to suppression of surface charging and provides a straightforward sample preparation strategy for MALDI MS imaging of tissues. An explanation for the signal enhancement in microprobe MALDI imaging may also be related to suppression of surface charging; however, other mechanisms cannot be excluded. Gold deposition on matrix crystals does not increase signal intensity or mass range in SIMS. The combination of MESIMS and MetA-SIMS is therefore not useful for tissue or single cell analysis.

We have shown that surface modification by applying either a matrix (ME-SIMS) or a layer of metal (MetA-SIMS and stigmatic MALDI-MS) results in enhanced intact molecule signals in imaging MS at the single-cell and tissue level. Although the mechanism of signal enhancement by gold deposition is under debate, the results we have obtained for cells and tissue sections illustrate the potential of this sample preparation technique for biomolecular surface imaging by mass spectrometry.

\section{ACKNOWLEDGMENT}

The authors thank Dr. Denitza Lambreva, for AFM measurements, Dr. Marcus Stoeckli, for communicating preliminary results of gold coating in microprobe imaging MALDI MS, and Bas Ponsioen and Michiel Langeslag for kindly supplying neuroblastoma cells. This work is part of research program 49 "Mass spectrometric imaging and structural analysis of biomacromolecules" of the "Stichting voor Fundamenteel Onderzoek der Materie (FOM)", which is financially supported by the "Nederlandse organisatie voor Wetenschappelijk Onderzoek (NWO)”.

Received for review July 22, 2005. Accepted November 18, 2005.

AC0513111 